\title{
Laryngopharyngeal dysfunction independent of vocal fold palsy in infants after aortic arch interventions
}

\author{
Ryan R. Davies, MD, ${ }^{\mathrm{a}, \mathrm{d}}$ Stephanie W. Carver, APN, ${ }^{\mathrm{a}}$ Richard Schmidt, MD,, $\mathrm{d}$ \\ Heather Keskeny, MA, CCC-SLP, ${ }^{c}$ Jeanine Hoch, MA, CCC-SLP, ${ }^{c}$ and Christian Pizarro, MD $^{\mathrm{a}, \mathrm{d}}$
}

\begin{abstract}
Objective: Laryngopharyngeal dysfunction contributes significantly to morbidity, length of stay, and increased resource utilization after aortic arch interventions in infants. Previous studies have focused on postoperative evaluation of selected symptomatic patients with vocal fold palsy (VFP). A prospective evaluation of laryngopharyngeal function was undertaken to assess and determine its importance in perioperative management.
\end{abstract}

Patients and methods: A routine preoperative and postoperative assessment was done by flexible fiber optic laryngoscopy, oral feeding evaluation (OFE), and modified barium swallow (mBS) on 101 infants undergoing 113 procedures on the aortic arch (2003 to 2011). The primary outcome was the ability to take full nutrition orally at discharge.

Results: Preoperative OFE was abnormal in $33.3 \%$ of patients. VFP occurred in $39.3 \%, 30$ of 57 patients had an abnormal OFE. Factors predictive of abnormal postoperative feeding evaluation included genetic syndromes (odds ratio [OR], 5.1; confidence interval [CI], 1.1-23.2) and preoperative mechanical ventilation (OR, 24.1; 95\% CI, 2.5-226.6). An abnormal postoperative OFE was highly predictive (OR, 7.0; 95\% $\mathrm{CI}, 1.8-27.1)$ of an abnormal postoperative $\mathrm{mBS}$. There was a trend toward decreased long-term survival among patients who were intubated or had an abnormal preoperative feeding evaluation $(P=.07)$.

Conclusions: Postoperative laryngopharyngeal dysfunction is common among infants undergoing aortic arch interventions, and is largely independent of vocal fold function. Preoperative evaluation demonstrates significant intrinsic abnormalities in sensory and motor function. Routine, comprehensive, multimodality preoperative and postoperative evaluation is required to identify at-risk children and reduce morbidity and resource utilization. (J Thorac Cardiovasc Surg 2014;148:617-24)

Supplemental material is available online.

Infants undergoing cardiovascular surgical procedures, particularly those involving the aortic arch, are at significant risk for laryngopharyngeal dysfunction (LPD) ${ }^{1-3}$ Aspiration resulting from disordered swallowing may result in significant morbidity and even mortality in high-risk infants. ${ }^{1}$ In addition, laryngopharyngeal dysfunction and nutritional difficulties may contribute to poor growth and long-term development in patients with congenital heart disease (CHD). ${ }^{4,5}$ Historically, most research has focused on vocal fold dysfunction as the

\footnotetext{
From the Nemours Cardiac Center, ${ }^{\mathrm{a}}$ Department of Otolaryngology, ${ }^{\mathrm{b}}$ and Division of Speech Pathology, ${ }^{\mathrm{c}}$ Nemours/A.I. duPont Hospital for Children, Wilmington, Del; and Thomas Jefferson University, ${ }^{\mathrm{d}}$ Philadelphia, Pa.

Disclosures: Authors have nothing to disclose with regard to commercial support.

Received for publication Sept 11, 2012; revisions received April 30, 2013; accepted for publication May 16, 2013; available ahead of print Dec 2, 2013.

Address for reprints: Ryan R. Davies, MD, Nemours/A.I. duPont Hospital for Children, 1600 Rockland Rd, Wilmington, DE 19803 (E-mail: rdavies@ nemours.org). $0022-5223 / \$ 36.00$

Copyright (c) 2014 by The American Association for Thoracic Surgery http://dx.doi.org/10.1016/j.jtcvs.2013.05.054
}

result of operative intervention and perioperative care. ${ }^{2,3,6}$ Although vocal fold dysfunction is an important contributor to LPD, many patients with poor feeding tolerance have normal vocal fold function. ${ }^{1}$ Whether feeding intolerance and swallowing abnormalities are the result of operative interventions or intrinsic sensory and motor dysfunction has not been assessed. Previous studies have included only postoperative assessments and have often limited evaluation to obviously symptomatic patients in the postoperative period. ${ }^{1-3,6}$ This article reports the results of a comprehensive preoperative and postoperative evaluation of oral feeding and laryngopharyngeal function in infants undergoing surgery on the aortic arch.

\section{PATIENTS AND METHODS \\ Patient Population}

Between 2004 and 2011, 101 patients underwent 113 aortic arch interventions at a single institution. These included stage 1 Norwood procedures (Norwood; $\mathrm{n}=34$ ), stage 1 hybrid Norwood procedures (Hybrid; $\mathrm{n}=16$ ), aortic arch augmentation for hypoplastic or interrupted aortic arch (Arch Augmentation; $\mathrm{n}=37$ ), repair of aortic coarctation (Aortic Coarctation; $\mathrm{n}=23$ ), and comprehensive stage 2 after a hybrid Norwood procedure (Comprehensive Stage 2; $\mathrm{n}=3$ ). During this period, a standardized prospective protocol was used for evaluation of oral feeding and laryngopharyngeal function. A retrospective review was conducted of all patients and risk factors for postoperative feeding difficulties were 


$$
\begin{aligned}
\text { Abbreviations and Acronyms } \\
\text { AUC }=\text { area under the curve } \\
\text { CHD }=\text { congenital heart disease } \\
\text { CI }=\text { confidence interval } \\
\text { CPB }=\text { cardiopulmonary bypass } \\
\text { DHCA }=\text { deep hypothermic circulatory arrest } \\
\text { FFOL }=\text { flexible fiber optic laryngroscopy } \\
\text { ICU }=\text { intensive care unit } \\
\text { LPD } \\
\text { mBS }=\text { moryngopharyngeal dysfunction } \\
\text { NPV }=\text { negative predictive value } \\
\text { OFE }=\text { oral feeding evaluation } \\
\text { OR }=\text { odds ratio } \\
\text { PAB }=\text { pulmonary artery band } \\
\text { PPV }=\text { positive predictive value } \\
\text { VFP }=\text { vocal fold palsy }
\end{aligned}
$$

investigated. The study was approved by the institutional investigational review board.

\section{Surgical Technique}

Surgical technique differed based on the procedure. Electrocautery was used for dissection. We routinely attempted to identify the recurrent laryngeal nerve; blunt retraction of the nerve and surrounding tissue was used as needed. Cardiopulmonary bypass (CPB) and deep hypothermic circulatory arrest (DHCA) were used at the surgeon's discretion. The decision to approach the arch via median sternotomy versus thoracotomy was made by the surgeon, taking into account additional lesions requiring $\mathrm{CPB}$ and the extent of the aortic arch reconstruction required. Transesophageal echocardiography was used sparingly and never in patients less than $3.5 \mathrm{~kg}$.

Aortic coarctation repair was performed with an extended end-to-end anastomosis via left thoracotomy. Aortic arch augmentation and stage I Norwood procedures were performed via median sternotomy using DHCA. Stage 1 hybrid Norwood procedures involved bilateral pulmonary artery band (PAB) placement and insertion of a ductal stent under fluoroscopy via median sternotomy. Where necessary for control of pulmonary blood flow, PAB placement was performed before ductal stenting; the timing of the hybrid intervention was considered to occur at completion of stage 1 hybrid Norwood by ductal stent insertion. Three patients underwent bilateral PAB followed by a traditional Norwood procedure (these patients are included in the Norwood group).

\section{Laryngopharyngeal Evaluation}

A standardized protocol for evaluation of laryngopharyngeal function was undertaken in all patients undergoing interventions on the aortic arch (Table E1). Evaluation included preoperative and postoperative flexible fiber optic laryngoscopy (FFOL) for evaluation of vocal fold palsy (VFP) by a single otolaryngologist, preoperative and postoperative oral feeding evaluation (OFE) by a selected group of speech therapists (Online Data Supplement 1), and postoperative modified barium swallow $(\mathrm{mBS})$ in selected patients. Patients requiring mechanical ventilation were not evaluated preoperatively. In addition, at the physicians' discretion, preoperative OFE was deferred when patients were at high risk for necrotizing enterocolitis. mBS was performed in selected patients: (1) those undergoing a complex aortic arch repair (Norwood, Arch Augmentation, Comprehensive Stage 2) or (2) those with an abnormality on FFOL or OFE. Evaluations of patients with multiple arch interventions were included in the analysis only when the evaluation occurred after an earlier intervention or before a subsequent intervention.

\section{Discharge, Follow-up, and Reevaluation}

Standardized criteria were used for discharge on enteral feeds by mouth. Patients had to have passed both OFE and mBS without evidence of aspiration. They had to tolerate a total of $120 \mathrm{~mL} / \mathrm{kg} / \mathrm{d}$ with weight gain on 3 consecutive days. In patients not tolerating full enteral feeds by mouth, tube feeding was used as appropriate. Patients with evidence of aspiration were reevaluated on a schedule (Table E1).

\section{Preoperative Risk Factors}

Preoperative risk factors were collected as defined in the Society of Thoracic Surgeons Congenital Heart Surgery Database. This included the presence of genetic syndromes and of any noncardiac congenital abnormality, as well as preoperative shock. Other risk factors analyzed included age, weight, prematurity, gender, diagnosis, a history of previous procedures, need for mechanical ventilation or admission to an intensive care unit, and operative variables including CPB and circulatory arrest times.

\section{Outcomes Measures}

The primary outcome was inability to tolerate oral feeds at discharge. Secondary outcomes included the occurrence of abnormalities on postoperative studies including FFOL, OFE, or mBS, overall incidence of complications, length of stay, hospital survival, and long-term survival. All examinations not demonstrating normal findings were considered abnormal. On FFOL, all examinations demonstrating abnormal vocal fold function are described as having VFP. It is our clinical practice to not attempt to distinguish between paralysis (complete loss of innervation) and paresis (partial loss of innervation) because, in babies, airflow dynamics and forces extrinsic to the larynx nearly always cause some passive movement of the vocal fold or arytenoids. It is nearly impossible to distinguish between such passive movement and movement due to residual innervation. Therefore, all abnormal FFOL examinations have been described here as demonstrating a VFP rather than paresis or paralysis. On OFE, failure to perform any of the components of the examination appropriately was considered an abnormal examination (Online Data Supplement 2). Laryngeal penetration, aspiration, and swallow discoordination present on mBS were all considered abnormal examinations.

\section{Statistical Methods and Data Analysis}

Statistical analysis was conducted using SAS 9.2 for AIX (SAS Institute Inc, Cary, NC). Statistical tests used to identify predictors of outcomes included the $\chi^{2}$ test and paired $t$ test. Results are reported as odds ratios (OR) and $95 \%$ confidence intervals (CI). When no patients in 1 group had an outcome of interest, ORs cannot be calculated and results are reported as frequency and $P$ value. The list of variables evaluated as risk factors is given in Table E2.

Longitudinal outcomes were assessed with Kaplan-Meier survival estimates (log-rank test for differences between strata, $P<.05$ ). Multivariate regression of binary outcomes was performed using logistic regression (backward selection, $P<.2$ selection).

To identify patients likely to benefit from particular examinations, logistic regression models were constructed (stepwise, $P<.04$ ) predicting the likelihood of an abnormal OFE or mBS using data available before the respective examination. Attempts were made to define a low-risk group in whom postoperative evaluations would not be required.

\section{RESULTS}

Baseline demographic and clinical variables are illustrated in Table 1. CPB and DHCA were used in all patients 
TABLE 1. Baseline demographics and clinical variables among infants undergoing arch interventions

\begin{tabular}{|c|c|}
\hline Variable & $\begin{array}{c}\text { Overall } \\
(n=113)\end{array}$ \\
\hline Age $(\mathrm{d}$, mean $\pm \mathrm{SD})$ & $34 \pm 62$ \\
\hline Weight $(\mathrm{kg}$, mean $\pm \mathrm{SD})$ & $3.4 \pm 1.0$ \\
\hline Body surface area $\left(\mathrm{m}^{2}\right.$, mean $\left.\pm \mathrm{SD}\right)$ & $0.22 \pm 0.04$ \\
\hline Gestational age (wk, mean \pm SD) & $37.9 \pm 1.9$ \\
\hline Birth weight $(\mathrm{kg}$, mean $\pm \mathrm{SD})$ & $3.0 \pm 0.6$ \\
\hline Gender (male), n (\%) & $54(47.8)$ \\
\hline Genetic syndrome, n (\%) & $21(19.3)$ \\
\hline Noncardiac congenital abnormality, n (\%) & $15(17.4)$ \\
\hline Premature, $\mathrm{n}(\%)$ & $15(13.4)$ \\
\hline \multicolumn{2}{|l|}{ Diagnosis } \\
\hline Normally related great arteries, n (\%) & $102(90.3)$ \\
\hline Hypoplastic left heart syndrome, n (\%) & 47 (41.6) \\
\hline $\begin{array}{l}\text { Hypoplastic aortic arch (without hypoplastic } \\
\text { left heart syndrome), } \mathrm{n}(\%)\end{array}$ & $35(30.0)$ \\
\hline Aortic coarctation, $\mathrm{n}(\%)$ & $26(23.0)$ \\
\hline Transposition of the great arteries, $\mathrm{n}(\%)$ & $6(5.3)$ \\
\hline $\begin{array}{l}\text { Totally anomalous pulmonary venous } \\
\text { connection, } \mathrm{n}(\%)\end{array}$ & $2(1.8)$ \\
\hline \multicolumn{2}{|l|}{ Preoperative variables } \\
\hline Previous sternotomy, n (\%) & $12(10.6)$ \\
\hline Previous palliative procedure, $\mathrm{n}(\%)$ & $14(15.1)$ \\
\hline Admitted to intensive care unit, $\mathrm{n}(\%)$ & $78(79.6)$ \\
\hline Mechanical ventilation, $\mathrm{n}(\%)$ & $24(21.2)$ \\
\hline Preoperative shock, $\mathrm{n}(\%)$ & $14(12.4)$ \\
\hline \multicolumn{2}{|l|}{ Operative variables } \\
\hline Cardiopulmonary bypass, $\mathrm{n}(\%)$ & $74(65.5)$ \\
\hline Time (min, mean \pm standard deviation) & $127.7 \pm 35.3$ \\
\hline Deep hypothermic circulatory arrest, $\mathrm{n}(\%)$ & $72(63.7)$ \\
\hline Time (min, mean \pm standard deviation) & $52.7 \pm 20.2$ \\
\hline Transesophageal echocardiography, n (\%) & $30(26.6)$ \\
\hline Aristotle Comprehensive Complexity Score & $13.0 \pm 6.1$ \\
\hline
\end{tabular}

$S D$, Standard deviation.

undergoing stage 1 Norwood or aortic arch augmentation, but not in patients undergoing stage 1 hybrid palliation or aortic coarctation repair.

\section{Preoperative Evaluation}

An abnormal preoperative FFOL occurred in 3 of 75 $(4.0 \%)$ examinations. These included 1 patient with a genetic syndrome (22q11 deletion and microcephaly), 1 patient with a history of prematurity but no genetic syndrome, and 1 patient with a previous arch operation. Preoperative OFE was performed in 48 patients, and was abnormal in 16 (Figure 1, A). Few $(\mathrm{n}=2)$ Hybrid patients could be evaluated because of mechanical ventilation or poor clinical status, but neither $(0.0 \%)$ had normal examinations compared with $69.5 \%$ for other patients $(P=.04)$. The risk of an abnormal preoperative examination was lowest among the Aortic Coarctation group ( 1 of $14,7.1 \% ; P=.01)$. Patients with genetic syndromes were at high risk of an abnormal preoperative examination (OR, 14.0; 95\% CI, 2.5-79.5).

\section{Postoperative Evaluation}

VFP. VFP was present in $44(39.3 \%)$ of 113 patients postoperatively; there were no differences based on the procedure (Norwood: 12 of 34, 35.3\%; Hybrid: 3 of 15, 20.0\%; Arch Augmentation: 17 of 36, 47.2\%; Aortic Coarctation: 11 of $24,45.8 \%$; Comprehensive Stage 2: 1 of $3,33.3 \% ; \quad P=$ not significant). The only univariate predictor of postoperative VFP was preoperative intensive care unit (ICU) admission (OR, 3.1; 95\% CI, 1.0-10.3).

OFE. OFEs were performed postoperatively in 97 patients (Figure 1, B). Among Hybrid patients, 9 did not have postoperative examinations ( $56.3 \%$ vs $7.2 \%$ for all others). Predictors of abnormal postoperative OFE are shown in Table 2. Patients with postoperative VFP were more likely to have an abnormal OFE (30 of 40 [75\%]; OR, 2.7; 95\% CI, 1.1-6.5), but 30 of 57 patients without VFP on FFOL $(52.6 \%)$ had an abnormal OFE.

mBS. Sixty-eight patients had a mBS. There were trends toward an increased incidence of abnormal mBS among patients with a previous arch intervention $(100 \%$ vs $77.1 \%, P=.15)$, a previous palliative procedure $(100 \%$ vs $76.0 \%, P=.12$ ), or an abnormal preoperative feeding evaluation (OR, 6.1; 95\% CI, 0.6-62.2; $P=.10$ ). An abnormal postoperative feeding evaluation (OR, 7.0; 95\% CI, 1.8-27.1) but not vocal cord palsy (OR, 1.3; 95\% CI, 0.4-4.1) was predictive of an abnormal mBS (Table 3).

Aspiration on mBS occurred in 44 patients $(64.7 \%)$; other abnormalities included: laryngeal penetration $(12,17.6 \%)$, reflux $(9,13.2 \%)$, and swallow discoordination $(8,11.8 \%)$. Patients intubated preoperatively were more likely to aspirate (OR, 2.7; 95\% CI, 1.1-6.8). Postoperatively, VFP was associated with aspiration on mBS (OR, 3.9; 95\% CI, 1.8-8.8), as was an abnormal OFE (OR, 4.0; 95\% CI, 1.6-10.2). Among patients without VFP, aspiration on mBS occurred in 16 of $31(51.6 \%)$ versus 25 of $37(67.6 \%)$ among patients with VFP. Presence of aspiration on feeding evaluation was also predictive of aspiration on $\mathrm{mBS}$ (OR, 3.9; 95\% CI, 1.2-12.3).

Among patients without aspiration on feeding evaluation, the negative predictive value (NPV) for aspiration on $\mathrm{mBS}$ was $64.2 \%$ (52 of 81 ), the positive predictive value (PPV) of aspiration on a feeding evaluation was $66.7 \%$ (10 of 15). Among patients with any abnormality noted on postoperative OFE, the PPV was $52.5 \%$ (31 of 59 ), and the NPV among those without any abnormality was $78.4 \%$ (29 of 37).

In multivariate analysis, patients with an abnormal postoperative OFE were at high risk for an abnormal mBS (OR, 4.6; 95\% CI, 1.0-19.8), whereas patients in the Aortic Coarctation group were less likely to have an abnormal mBS (OR, 0.2; 95\% CI, 0.0-1.1).

Modeling of likelihood of abnormal examinations. A model highly predictive of an abnormal postoperative OFE 


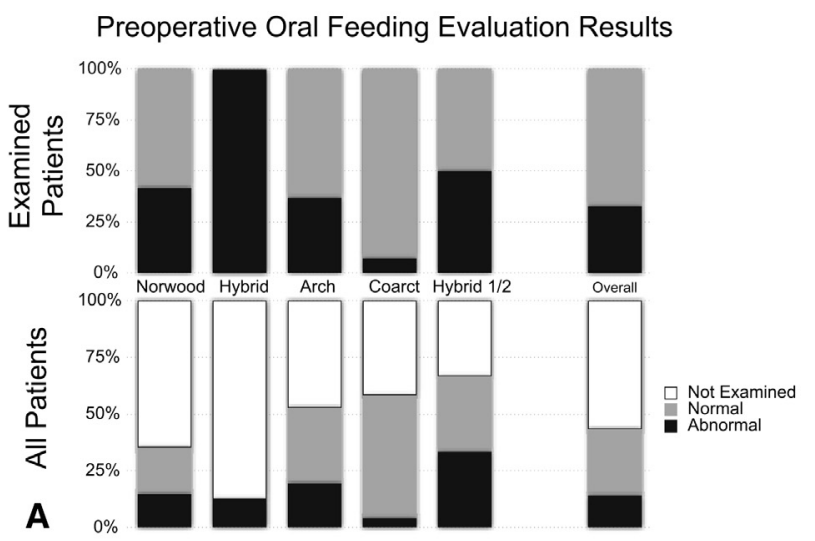

Postoperative Oral Feeding Evaluation Results
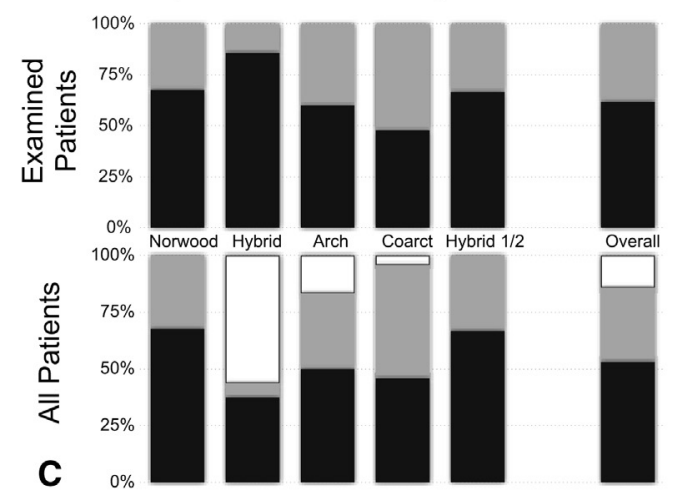

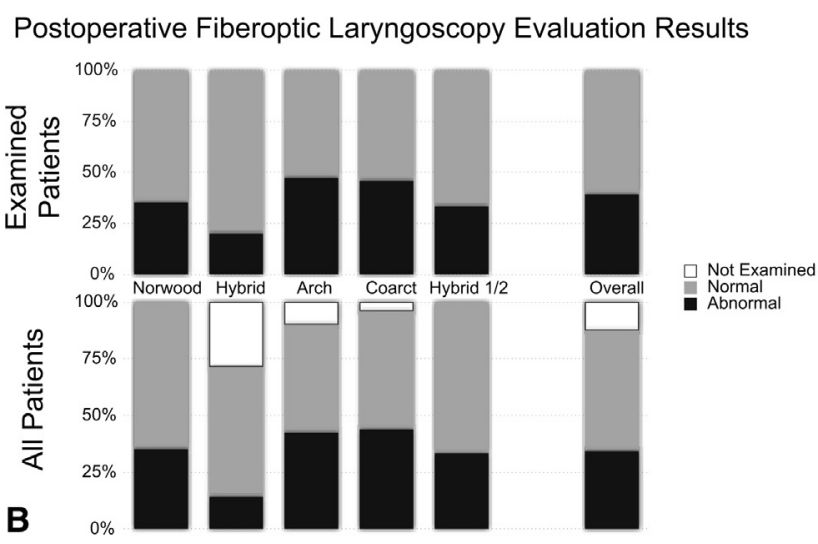

Postoperative Modified Barium Swallow

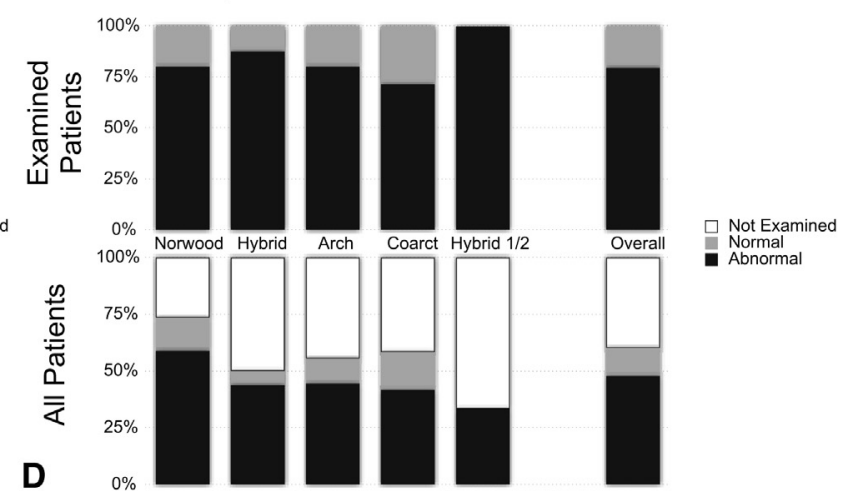

FIGURE 1. A, Preoperative oral feeding evaluations; B, postoperative fiber optic laryngoscopy; C, postoperative oral feeding evaluation; D, postoperative modified barium swallow. In each section, the lower half of the graph demonstrates all patients including those not evaluated (white), normal (gray), and abnormal (black) examinations. The upper half illustrates only the patients undergoing the specified evaluation.

was developed (area under the curve [AUC], 0.9208; Table 3), but prediction of an abnormal mBS was less robust (AUC, $0.7316)$ and included only 2 variables: aortic coarctation surgery (OR, $0.21 ; 95 \% \mathrm{CI}, 0.04-1.14$ ) and an abnormal postoperative OFE (OR, 4.6; 95\% CI 1.1-19.8). Using this model, no subset of patients could be identified in which the risk of an abnormal postoperative examination was 0 .

\section{Outcomes}

Postoperative feeding. Patients were less likely to be discharged tolerating oral feeding after hybrid procedures (Norwood: 15 of 34, 44.1\%; Hybrid: 1 of 16, 6.3\%; Arch Augmentation: 23 of 36, 63.9\%; Aortic Coarctation: 19 of $24,79.2 \%$; Comprehensive Stage 2: 0 of 3, $0.0 \%$ : $P<.0001$ ) (Table E3). Univariate predictors of the inability to tolerate full enteral feeds are shown in Table 3.

Patients able to tolerate full oral feeds were larger at surgery (3.4 vs $2.9 \mathrm{~kg} ; P=.0097$ ), had lower Aristotle Comprehensive Complexity Scores (10.6 vs 14.4; $P=.0011)$, shorter times to extubation (219.3 vs 67.6 hours; $P=.0059)$, and shorter ICU stay (7.2 vs 22.6 days; $P=.0005)$ and overall stay (18.6 vs 44.6 days; $P<.0001)$.

A multivariate model of preoperative factors predicting the inability to tolerate full oral intake included the following variables: abnormal preoperative OFE (OR, 20.6; 95\% CI, 1.9-226.1), redo arch intervention (OR, 18.5; 95\% CI, 1.2-281.5), preoperative tracheal intubation (OR, 4.6; 95\% CI, 1.0-20.0), preoperative OFE not performed (OR, 2.3; 95\% CI, 0.8-7.2), age at surgery (OR, 1.009 per day; 95\% CI, 0.995-1.024), diagnosis of hypoplastic aortic arch (OR, 0.5; 95\% CI, 0.2-1.4), diagnosis of isolated aortic coarctation (OR, $0.4 ; 95 \% \mathrm{CI}$, 0.1-1.3), and weight (OR, 0.2 per $\mathrm{kg} ; 95 \% \mathrm{CI}, 0.1-0.7$ ). AUC for this model was 0.8374 , indicating good predictive ability using preoperative factors only (Figure E1). The addition of intra- and postoperative data (excluding OFE and mBS results) improved the model only slightly (Table 3; AUC, 0.8562); this revised model included ICU length of stay (OR, 1.136 per day; 95\% CI, 1.04-1.24).

Surgical gastrostomy tubes were placed in 27 patients. Patients undergoing hybrid procedures were more likely to have a gastrostomy tube (Comprehensive Stage 2: 66.7\%, Hybrid: $57.1 \%$, Norwood: $27.6 \%$, Arch Augmentation: $31.5 \%$, Aortic Coarctation: $8.7 \% ; P=.05)$. Nissen fundoplications were performed in 26 patients.

Among patients not tolerating full oral feeds at discharge, 32 $(45.1 \%)$ eventually recovered function during the study 
TABLE 2. Significant predictors of abnormal postoperative oral feeding evaluations and abnormal postoperative modified barium swallow in both univariate $(P<.10)$ and multivariate $(P<.20)$ analysis*

\begin{tabular}{|c|c|c|c|c|c|c|}
\hline \multirow[b]{2}{*}{ Risk factor } & \multicolumn{2}{|c|}{$\begin{array}{c}\text { Abnormal oral } \\
\text { feeding evaluation }\end{array}$} & \multicolumn{2}{|c|}{$\begin{array}{l}\text { Abnormal modified } \\
\text { barium swallow }\end{array}$} & \multicolumn{2}{|c|}{$\begin{array}{c}\text { Inability to tolerate } \\
\text { full postoperative feeds }\end{array}$} \\
\hline & $\begin{array}{c}\text { Univariate } \\
\text { analysis, } \\
\text { OR }(95 \% \text { CI }) \\
\end{array}$ & $\begin{array}{c}\text { Multivariate } \\
\text { analysis, } \\
\text { OR }(95 \% \text { CI }) \\
\end{array}$ & $\begin{array}{c}\text { Univariate } \\
\text { analysis, } \\
\text { OR }(95 \% \mathrm{CI}) \\
\end{array}$ & $\begin{array}{c}\text { Multivariate } \\
\text { analysis, } \\
\text { OR }(95 \% \mathrm{CI}) \\
\end{array}$ & $\begin{array}{c}\text { Univariate } \\
\text { analysis, } \\
\text { OR }(95 \% \text { CI }) \\
\end{array}$ & $\begin{array}{c}\text { Multivariate } \\
\text { analysis, } \\
\text { OR }(\mathbf{9 5} \% \mathbf{C I}) \ddagger \\
\end{array}$ \\
\hline \multicolumn{7}{|l|}{ Preoperative clinical status } \\
\hline Weight (per additional kg) & - & $0.3(0.1-0.9)$ & - & - & $0.6(0.4-0.9)$ & $0.4(0.0 .2-0.9)$ \\
\hline Height (per additional $\mathrm{cm}$ ) & & & & & $0.9(0.9-1.0)$ & - \\
\hline $\begin{array}{l}\text { Body surface area } \\
\quad\left(\text { per additional } \mathrm{m}^{2}\right)\end{array}$ & & & & & $0.001(0.001-0.2)$ & - \\
\hline Genetic syndrome & $4.2(1.1-15.6)$ & - & - & - & $2.8(0.9-9.0)$ & $3.0(0.6-14.3)$ \\
\hline $\begin{array}{l}\text { Other noncardiac } \\
\text { abnormality }\end{array}$ & - & & - & - & $7.6(0.9-61.4)$ & - \\
\hline Prematurity (<37 wk) & - & & - & - & - & - \\
\hline $\begin{array}{l}\text { Preoperative mechanical } \\
\text { ventilation }\end{array}$ & $14.2(1.8-112.5)$ & $21.1(1.5-284.0)$ & - & - & $5.5(1.5-19.6)$ & $7.5(1.8-31.5)$ \\
\hline Preoperative shock & $6.4(0.8-52.4)$ & - & - & - & $4.1(0.9-19.2)$ & - \\
\hline $\begin{array}{l}\text { Preoperative admission to } \\
\text { intensive care unit }\end{array}$ & - & $14.7(1.1-196.3)$ & - & - & $3.4(1.2-9.3)$ & - \\
\hline \multicolumn{7}{|l|}{ Congenital cardiac diagnosis } \\
\hline $\begin{array}{l}\text { Hypoplastic left heart } \\
\text { syndrome }\end{array}$ & - & & - & - & $2.9(1.3-6.7)$ & - \\
\hline $\begin{array}{l}\text { Hypoplastic aortic arch } \\
\text { ( } 2 \text { ventricles })\end{array}$ & - & $0.2(0.0-0.8)$ & - & - & $0.5(0.2-1.1)$ & $0.4(0.1-1.3)$ \\
\hline Isolated aortic coarctation & - & & - & $0.2(0.0-1.1)$ & $0.4(0.2-1.0)$ & $0.3(0.1-1.0)$ \\
\hline \multicolumn{7}{|l|}{ Procedures } \\
\hline Hybrid procedure & $4.0(0.5-34.6)$ & - & - & - & Relative risk $(1.4,1.2-1.6) \dagger$ & - \\
\hline $\begin{array}{l}\text { Isolated aortic coarctation } \\
\text { repair }\end{array}$ & - & $0.2(0.0-1.4)$ & - & - & - & - \\
\hline $\begin{array}{l}\text { Reoperative aortic arch } \\
\text { intervention }\end{array}$ & - & $10.8(0.3-291.4)$ & - & - & - & $130.0(4.0-999.9)$ \\
\hline $\begin{array}{l}\text { Aortic crossclamp time } \\
\quad \text { (per additional minute) }\end{array}$ & - & - & - & - & $0.99(0.97-1.00)$ & \\
\hline \multicolumn{7}{|l|}{ Preoperative examinations } \\
\hline $\begin{array}{l}\text { Abnormal preoperative } \\
\text { oral feeding evaluation }\end{array}$ & $35.8(4.0-319.3)$ & $35.2(2.0-61.6)$ & $6.1(0.6-62.0)$ & $4.6(1.1-19.8)$ & $11.7(2.3-60.5)$ & $16.2(1.4-192.9)$ \\
\hline $\begin{array}{l}\text { Preoperative oral feeding } \\
\text { evaluation not performed }\end{array}$ & - & $10.2(1.5-65.5)$ & - & - & $1.9(0.9-4.1)$ & - \\
\hline \multicolumn{7}{|l|}{ Postoperative examinations } \\
\hline $\begin{array}{l}\text { Postoperative vocal fold } \\
\text { palsy }\end{array}$ & $2.7(1.1-6.5)$ & $4.6(1.0-21.2)$ & - & - & - & - \\
\hline $\begin{array}{l}\text { Abnormal postoperative } \\
\text { oral feeding evaluation }\end{array}$ & - & - & $7.0(1.8-27.1)$ & - & $21.3(7.4-62.3)$ & - \\
\hline $\begin{array}{l}\text { Abnormal postoperative } \\
\text { modified barium } \\
\text { swallow }\end{array}$ & - & - & - & - & $6.7(1.9-23.9)$ & - \\
\hline
\end{tabular}

Isolated aortic coarctation

solated aortic coarctation

eoperative aortic arch

ortic crossclamp time

operative examinations

bnormal preoperative

reoperative oral feeding

evaluation not performe

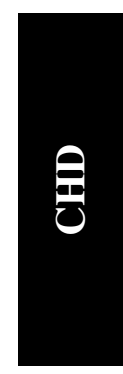


TABLE 3. Model predicting the likelihood of abnormal postoperative oral feeding evaluation using variables available before the examination (model fitting parameters: area under the curve, 0.9208; Hosmer-Lemeshow test, $P=.1671$ )

\begin{tabular}{|c|c|c|c|c|}
\hline & Odds ratio & $\mathbf{9 5} \%$ confidence interval & Parameter estimate & Standard error \\
\hline Model intercept & - & - & 1.1770 & 1.8374 \\
\hline Diagnosis: hypoplastic aortic arch (2 ventricles) & 0.27 & $0.07-1.08$ & -1.3284 & 0.7186 \\
\hline Surgery: aortic coarctation & 0.32 & $0.05-2.24$ & -1.1336 & 0.9895 \\
\hline Weight (per kg) & 0.43 & $0.15-1.20$ & -0.8467 & 0.5271 \\
\hline Gender: female & 1.85 & $0.45-7.58$ & 0.3068 & 0.3601 \\
\hline Postoperative vocal fold palsy & 3.1 & $0.8-12.2$ & 1.1157 & 0.7078 \\
\hline Preoperative oral feeding evaluation could not be performed & 6.0 & $1.2-30.5$ & 1.7950 & 0.8287 \\
\hline Preoperative mechanical ventilation & 18.0 & $1.5-212.8$ & 2.8920 & 1.2594 \\
\hline Abnormal preoperative oral feeding evaluation & 18.8 & $1.4-262.0$ & 2.9329 & 1.3447 \\
\hline Reoperative arch intervention & 20.0 & $0.9-483.9$ & 2.9972 & 1.6248 \\
\hline
\end{tabular}

period. Recovery of function occurred at a median of 46.5 days after discharge (interquartile range, 10.5-120 days). None of the preoperative or postoperative examinations were predictive of the time to recovery of function.

Mortality. In 5 patients (all with hypoplastic left heart syndrome; 5 of $47, P=.0067$ vs other diagnoses), death occurred before hospital discharge. Four of these patients had undergone hybrid Norwood procedures (OR, 32.0; 95\% CI, 3.3-310.4). Multivariate modeling of death before hospital discharge included the following factors: weight (OR, 0.099 per kg; 95\% CI, 0.012-0.835; $P=.03$ ), preoperative shock (OR, 31.8; 95\% CI, 2.4-421.1; $P<.009$ ), and an abnormal preoperative OFE (OR, 7.4; 95\% CI, 0.3-127.1; $P=.17$ ).

Eleven patients (11 of 113,10.2\%) died during long-term follow-up. Preoperative OFE was not predictive of long-term survival (Figure 2); there was a trend toward poorer survival among patients who were either intubated or had an abnormal preoperative examination (Figure 2).

\section{DISCUSSION}

Interventions on the aortic arch are common pediatric and infant surgical procedures in patients with CHD. Among patients less than 1 year old, procedures involving aortic arch anomalies account for more than $25 \%$ of operations within the Society of Thoracic Surgeons Congenital Heart Surgery Database. ${ }^{7}$ Although the risk of mortality from these and all other pediatric cardiac surgery procedures has decreased since the early 1990s, significant morbidity remains. ${ }^{8}$ Long-term sequelae, including neurodevelopmental problems, respiratory complications, and somatic growth restrictions, may have significant effects. ${ }^{9-12}$ In addition, the sequelae may have significant consequences in terms of length of stay and hospital costs. ${ }^{13,14}$

Nutritional difficulties in particular may result in both shortterm increases in length of stay and in longer-term alterations to somatic growth potential. ${ }^{1,15}$ Unfortunately, infants who have undergone cardiac surgery may have a wide range of problems resulting in the inability to tolerate oral feeds. ${ }^{1}$ The relative influence of preoperative sensory and motor dysfunction, operative technique, and perioperative interventions including transesophageal echocardiogram and duration of mechanical ventilation has not been assessed. ${ }^{1,16}$ Relying solely on postoperative examinations may conflate these issues without accounting for factors present even before operative intervention.

This article reports our experience with routine preoperative and postoperative examinations of vocal fold and oropharyngeal function among infants undergoing aortic arch surgery. The data suggest that laryngopharyngeal function in infants is far more complicated than previous studies would support, involving sensory and motor capabilities, vocal fold function, and overall clinical condition. Furthermore, preoperative assessment is highly predictive of both postoperative function and morbidity and mortality.

Although preoperative laryngoscopy did not identify a significant incidence of abnormal vocal fold function, preoperative OFEs did reveal a high incidence of preoperative motor and sensory dysfunction in these patients. Not all patients could be evaluated, but even among those who were, the incidence of an abnormal preoperative examination was $33.3 \%$. If anything, this represents an underestimation of the true incidence because patients at highest risk (mechanically ventilated or in poor clinical status) could not be evaluated.

In our series, patients with genetic syndromes were at particularly high risk of having preoperative swallowing dysfunction; only $20 \%$ passed the swallowing evaluation. Routine evaluation of these patients may help with postoperative planning for both the hospital care team and parents regarding expectations and appropriate management. We found that a higher Aristotle Comprehensive Complexity Score was associated with an increased risk for abnormal preoperative function. As this score includes features indicating both procedural (and relatedly diagnostic) complexity as well as preoperative condition, we believe it indicates that multiple factors including diagnosis, syndromic history, and clinical condition may play a role in preoperative dysfunction. In contrast, patients who were not hospitalized in the ICU and those undergoing 


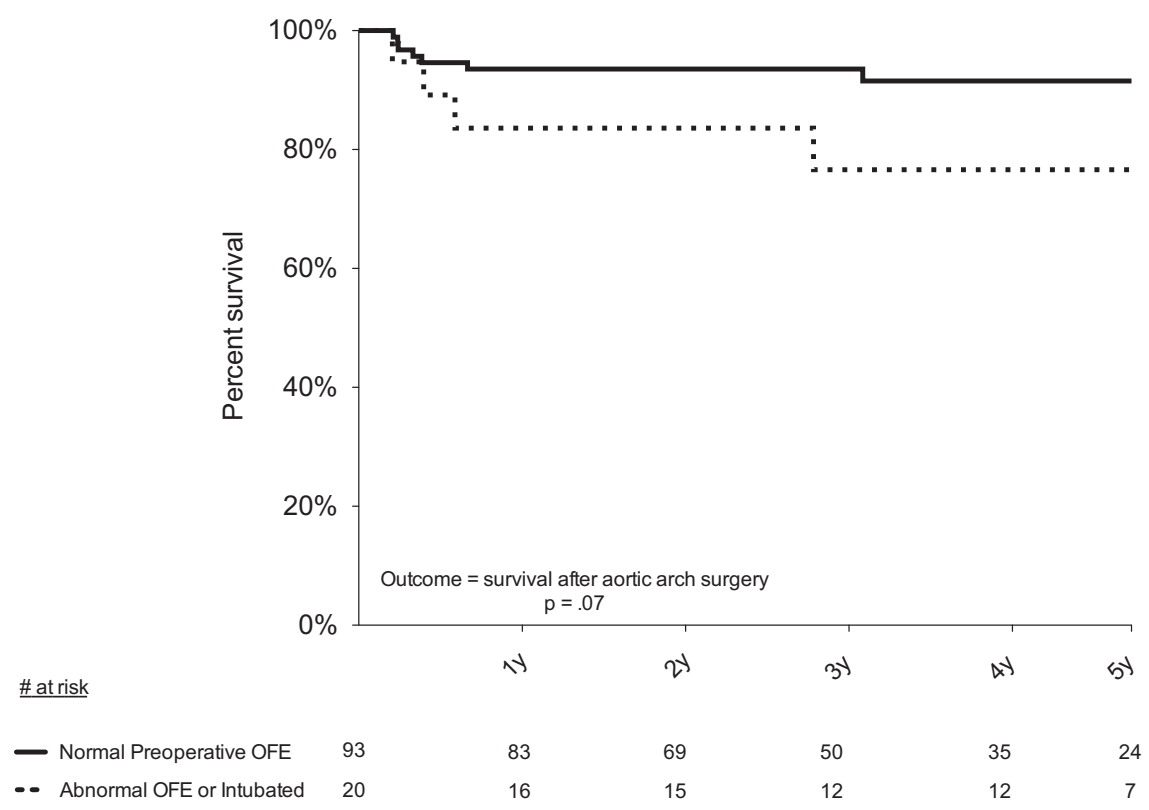

FIGURE 2. Kaplan-Meier survival estimates after surgical repair. A, Patients are stratified by the findings on preoperative oral feeding evaluation (OFE) into 2 groups. Group 1 includes patients who had an abnormal examination or were intubated preoperatively (dotted line). Group 2 includes those patients who were not intubated and had a normal preoperative examination (solid line) $(P=.07)$.

isolated aortic coarctation repair were at very low risk for preoperative dysfunction.

Vocal fold dysfunction is commonly described as a complication of aortic arch surgery in children, ${ }^{2,17,18}$ and has traditionally been identified as the primary antecedent of oropharyngeal dysfunction and aspiration. ${ }^{3,16}$ In the absence of previous surgery, the incidence of preoperative VFP is negligible and related primarily to genetic syndromes. Routine preoperative evaluation of the vocal cords in the absence of either previous arch intervention or a genetic syndrome is probably not valuable.

The incidence of postoperative VFP was higher here than in previous studies, whether looking at the more complex Norwood procedures or aortic coarctation via thoracotomy. ${ }^{1,18}$ This may be partially reflective of the routine nature of our examinations. In comparison, symptom-directed vocal fold evaluation is likely to miss some patients with abnormalities because patients with VFP may be asymptomatic (as described both here and by others). ${ }^{1}$ In addition, we used palsy rather than paralysis to define an abnormal FFOL. This decision was made in part because of the difficulty in reliably differentiating paralysis from paresis on FFOL, and in part to assess whether this broader definition of vocal fold dysfunction would have additional predictive ability over previous studies. ${ }^{1}$ This was not the case.

Our rate of VFP after aortic coarctation repair via thoracotomy was high $(45 \%)$. Although this rate seems high, no comparative data exist. Carpes and colleagues ${ }^{18}$ found a lower rate of vocal fold paralysis (3 of 19) among aortic arch procedures; palsy seems to be more common. Despite this rate, most patients undergoing aortic coarctation repair were discharged on full oral nutrition, again supporting the idea that intrinsic factors and not simply vocal fold function are critical in determining laryngopharyngeal function in these patients.

Consistent with Skinner and colleagues, ${ }^{1}$ we have found that although vocal fold dysfunction is associated with swallowing abnormalities in patients undergoing the Norwood procedure, it is neither necessary nor sufficient to explain laryngopharyngeal dysfunction and oral feeding difficulties. More than half of the patients with abnormal feeding evaluations had normal vocal fold function. Conversely, $25 \%$ of patients with abnormal vocal folds had no consequent swallowing dysfunction. This suggests that evaluation of laryngopharyngeal function in these patients should not be limited to laryngoscopy.

Similarly, OFE cannot be consider the gold standard. Thirty patients demonstrated silent aspiration on $\mathrm{mBS}$; that is, despite a normal feeding evaluation by a trained speech pathologist, aspiration events were still noted on radiographic examinations. Five patients with aspiration on an OFE had subsequent mBS examinations that failed to demonstrate aspiration. Thus, it is critical to use these examinations in a complementary fashion. No single examination was capable of identifying all at-risk children; routine multimodality testing is essential.

Although multivariate modeling demonstrated significant predictors of risk for abnormal examinations and high-risk groups could be identified, in no group was the 
risk 0 . This suggests that routine examinations are required to identify all patients with disordered swallowing after aortic arch interventions.

Our data confirm the findings from Skinner and colleagues, ${ }^{1}$ that patients unable to tolerate oral feeds have longer hospital stays. There are several possible mechanisms for this. Patients with a single ventricle with risk for aspiration are less likely to be discharged home given our concern for aspiration and rapid deterioration in an unmonitored environment. In addition, given that we use toleration of enteral (although not necessarily oral) feeding as a prerequisite for discharge, it is inevitable that patients unable to tolerate feeds will stay longer.

In the long term, most patients recover swallowing function, although at times that may take more than 1 year. Ongoing follow-up with training to manage disordered swallowing as patients become older is important. Among patients with single-ventricle physiology and others at higher risk from aspiration, close monitoring of patients with disordered swallowing is critical to prevent interstage mortality, especially after hospital discharge. Long-term mortality was higher among patients with swallowing difficulties. This was most notable among patients with abnormal preoperative feeding evaluations, again demonstrating the lasting effect of intrinsic patient characteristics such as genetic syndromes on both swallowing function and long-term mortality.

\section{Limitations}

Several limitations of this study should be enumerated. It was a retrospective study of a population at a single institution. Although consonant with others' findings, further examination of the usefulness and cost-effectiveness of routine examinations at other institutions is warranted. It is difficult to establish the causal link and direction in many of these associations. For example, is a longer length of stay in the ICU the result of poor feeding and aspiration or the cause. Because of the limited sample size, important, clinically relevant associations may have been not met statistical significance, and we can only speculate regarding a link between oropharyngeal dysfunction, aspiration events, and interstage mortality in children with a single ventricle.

\section{CONCLUSIONS}

These data demonstrate that postoperative laryngopharyngeal function in infants undergoing aortic arch surgery is more complicated than the presence or absence of vocal fold dysfunction. It is a multifactorial process influenced by both intrinsic patient characteristics and perioperative care. Preoperative evaluation demonstrates that at least one-third of patients have disorders in sensory and motor aspects of swallowing. The incidence is even higher among patients with genetic syndromes. Postoperatively, asymptomatic patients without clinical evidence of vocal fold dysfunction may still aspirate, and no single postoperative examination identified all patients at risk for aspiration. Prediction of a low-risk group not needing postoperative examination was not possible. Routine, comprehensive, multimodality evaluation of laryngopharyngeal dysfunction among patients undergoing arch interventions is essential to minimize morbidity and mortality in this at-risk population.

\section{References}

1. Skinner ML, Halstead LA, Rubinstein CS, Atz AM, Andrews D, Bradley SM Laryngopharyngeal dysfunction after the Norwood procedure. J Thorac Cardiov Surg. 2005; 130:1293-301.

2. Truong MT, Messner AH, Kerschner JE, Scholes M, Wong-Dominguez J, Milczuk HA, et al. Pediatric vocal fold paralysis after cardiac surgery: rate of recovery and sequelae. Otolaryngol Head Neck Surg. 2007;137:780-4.

3. Sachdeva R, Hussain E, Moss MM, Schmitz ML, Ray RM, Imamura M, et al. Vocal cord dysfunction and feeding difficulties after pediatric cardiovascular surgery. J Pediatr. 2007;151:312-5, 315.e1-2.

4. Williams RV, Zak V, Ravishankar C, Altmann K, Anderson J, Atz AM, et al. Factors affecting growth in infants with single ventricle physiology: a report from the Pediatric Heart Network Infant Single Ventricle Trial. J Pediatr. 2011;159:1017-22.e2.

5. Anderson JB, Marino BS, Irving SY, García-España JF, Ravishankar C, Stallings VA, et al. Poor post-operative growth in infants with two-ventricle physiology. Cardiol Young. 2011;21:421-9.

6. Clement WA, EI-Hakim H, Phillipos EZ, Coté JJ. Unilateral vocal cord paralysis following patent ductus arteriosus ligation in extremely low-birth-weight infants. Arch Otolaryngol Head Neck Surg. 2008;134:28-33.

7. Society of Thoracic Surgeons. STS Congenital Heart Surgery Database. Available at: http://www.sts.org/sts-national-database/database-managers/ executive-summaries. Accessed May 2012.

8. Kaltman JR, Andropoulos DB, Checchia PA, Gaynor JW, Hoffman TM, Laussen PC, et al. Report of the pediatric heart network and national heart, lung, and blood institute working group on the perioperative management of congenital heart disease. Circulation. 2010;121:2766-72.

9. Miatton M, De Wolf D, François K, Thiery E, Vingerhoets G. Neuropsychological performance in school-aged children with surgically corrected congenital heart disease. J Pediatr. 2007;151:73-8, 78.e1.

10. Bellinger DC, Wypij D, Rivkin MJ, DeMaso DR, Robertson RL, DunbarMasterson C, et al. Adolescents with d-transposition of the great arteries corrected with the arterial switch procedure: neuropsychological assessment and structural brain imaging. Circulation. 2011;124:1361-9.

11. Schuurmans FM, Pulles-Heintzberger CF, Gerver WJ, Kester AD, Forget PP. Long-term growth of children with congenital heart disease: a retrospective study. Acta Paediatr. 1998;87:1250-5.

12. Polat S, Okuyaz C, Hallioğlu O, Mert E, Makharoblidze K. Evaluation of growth and neurodevelopment in children with congenital heart disease. Pediatr Int. 2011;53:345-9.

13. Benavidez OJ, Connor JA, Gauvreau K, Jenkins KJ. The contribution of complications to high resource utilization during congenital heart surgery admissions. Congenit Heart Dis. 2007;2:319-26.

14. Pasquali SK, Li JS, Burstein DS, Sheng S, O’Brien SM, Jacobs ML, et al. Association of center volume with mortality and complications in pediatric heart surgery. Pediatrics. 2012;129:e370-6.

15. Cribbs RK, Heiss KF, Clabby ML, Wulkan ML. Gastric fundoplication is effective in promoting weight gain in children with severe congenital heart defects. J Pediatr Surg. 2008;43:283-9.

16. Kohr LM, Dargan M, Hague A, Nelson SP, Duffy E, Backer CL, et al. The incidence of dysphagia in pediatric patients after open heart procedures with transesophageal echocardiography. Ann Thorac Surg. 2003;76:1450-6.

17. Malcolm WF, Hornik C, Evans A, Smith PB, Cotten CM. Vocal fold paralysis following surgical ductal closure in extremely low birth weight infants: a case series of feeding and respiratory complications. J Perinatol. 2008;28: 782-5.

18. Carpes LF, Kozak FK, Leblanc JG, Campbell AI, Human DG, Fandino M, et al. Assessment of vocal fold mobility before and after cardiothoracic surgery in children. Arch Otolaryngol Head Neck Surg. 2011;137:571-5. 


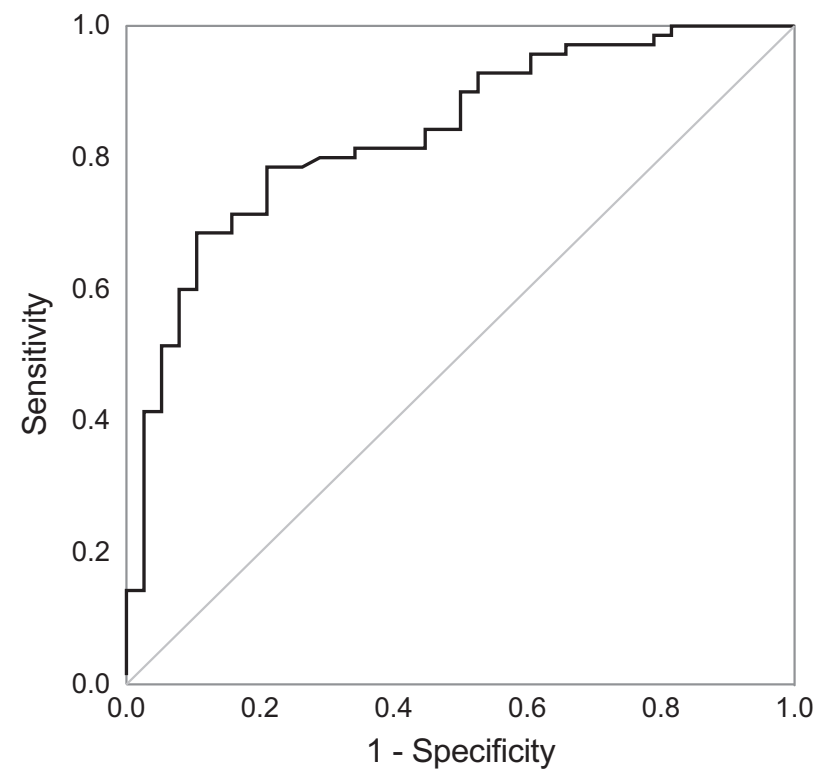

FIGURE E1. Receiver-operator curve $(R O C)$ of the model predicting inability to tolerate full oral feeds at discharge using only preoperative variables.

TABLE E1. Standardized preoperative and postoperative feeding evaluation

Vocal fold evaluation

Oral pharyngeal feeding evaluation

Modified barium swallow
Preoperative vocal fold evaluation performed on extubated patients with the following diagnoses: hypoplastic left heart syndrome; interrupted aortic arch; coarctation of the aorta; aortic arch hypoplasia; other congenital cardiovascular defects which, when surgically palliated, may involve injury to the recurrent laryngeal nerve

Postoperative vocal fold evaluation when the trachea is extubated and before the initiation of oral feeds (performed before other evaluations)

If postoperative vocal fold function is normal, no further reevaluation

If vocal fold palsy is present, reevaluation to occur at 1 month, 3 months, and every subsequent 3 months until resolution

Preoperative and postoperative oral pharyngeal feeding evaluation with speech therapy (see Online Data Supplement 2)

Modified barium swallow performed selectively on postoperative patients who (1) underwent a complex aortic arch repair (Norwood, Arch Augmentation, Comprehensive Stage 2) or (2) had an abnormal FFOL or OFE

If abnormal swallow study and abnormal vocal fold evaluation, reevaluation in 1 month

If abnormal swallow study and normal vocal fold evaluation, reevaluation in 3 months (unless taking thickened oral feeds in which earlier evaluation may be performed). If abnormal swallow study persists, reevaluate every 3 months for 1 year, then 6 months, then yearly 
TABLE E2. List of variables evaluated as risk factors for primary and secondary outcomes

\begin{tabular}{lc}
\hline Preoperative variables & $\begin{array}{c}\text { Operative/postoperative } \\
\text { variables }\end{array}$ \\
\hline Preoperative clinical status & Procedures \\
Gender (male) & Hybrid procedure \\
Age (per d) & Isolated aortic coarctation repair \\
Weight (per additional kg) & Previous palliative procedure \\
Height (per additional cm) & Reoperative aortic arch \\
& intervention \\
Body surface area & Cardiopulmonary bypass \\
(per additional $\mathrm{m}^{2}$ ) & Deep hypothermic circulatory \\
Genetic syndrome & arrest \\
& Aortic crossclamp time \\
Other noncardiac abnormality & (per additional min) \\
Prematurity (<37 wk) & Use of transesophageal \\
Preoperative mechanical & echocardiography \\
ventilation & Aristotle Comprehensive \\
Preoperative shock & Complexity Score \\
Preoperative admission to & Preoperative examinations \\
intensive care unit & Preoperative vocal fold palsy \\
Congenital cardiac diagnosis & Preoperative flexible fiber optic \\
Hypoplastic left heart syndrome & laryngroscopy not performed \\
Hypoplastic aortic arch & Abnormal preoperative oral \\
(2 ventricles) & feeding evaluation \\
Isolated aortic coarctation & Preoperative oral feeding \\
& evaluation not performed \\
& Postoperative examinations \\
& Postoperative vocal fold palsy \\
& Abnormal postoperative oral \\
feeding evaluation
\end{tabular}

TABLE E3. Postoperative feeding among patients undergoing arch surgery*

\begin{tabular}{|c|c|c|c|c|c|c|c|}
\hline & $\begin{array}{c}\text { Norwood } \\
(\mathrm{n}=\mathbf{3 4})\end{array}$ & $\begin{array}{c}\text { Hybrid } \\
(\mathrm{n}=16)\end{array}$ & $\begin{array}{c}\text { Arch } \\
(\mathbf{n}=36)\end{array}$ & $\begin{array}{c}\text { Coarctation } \\
(\mathrm{n}=\mathbf{2 4})\end{array}$ & $\begin{array}{l}\text { Hybrid } \\
(\mathbf{n}=\mathbf{3}) \\
\end{array}$ & $\begin{array}{c}P \\
\text { value }\end{array}$ & Overall \\
\hline Full oral feeds, n (\%) & $11(32.4)$ & 0 of $9(0.0)$ & 16 of $34(47.1)$ & $15(62.5)$ & $0(0.0)$ & $.006 \dagger$ & 42 of $113(37.2)$ \\
\hline Oral + tube feeds, $\mathrm{n}(\%)$ & $4(11.8)$ & 1 of $9(11.1)$ & 7 of $34(25.0)$ & $4(16.7)$ & $0(0.0)$ & & 11 of $113(9.7)$ \\
\hline Completely tube feeding, $\mathrm{n}(\%)$ & $19(55.9)$ & 8 of $9(88.9)$ & 11 of $34(30.5)$ & $5(20.8)$ & $3(100.0)$ & & 29 of $113(25.7)$ \\
\hline Gastrostomy tube feeding, $\mathrm{n}(\%)$ & $4(11.8)$ & 3 of $16(18.8)$ & 9 of $36(25.0)$ & $2(8.3)$ & $2(66.7)$ & .07 & 20 of $113(17.7)$ \\
\hline
\end{tabular}

